\title{
Examples of Discrete Schrödinger Operators with Pure Point Spectrum
}

\author{
Jürgen Pöschel \\ Mathematik, ETH-Zentrum, CH-8092 Zürich, Switzerland
}

\begin{abstract}
We present a general approach for constructing potentials for the discrete Schrödinger equation of arbitrary dimension having only pure point spectrum. We give examples of limit periodic potentials of that kind such that the pure point spectrum is dense in an interval or a Cantor set of measure zero.
\end{abstract}

\section{Introduction}

In this note we consider the discrete Schrödinger operator

$$
(H u)_{i}=\varepsilon \sum_{|\ell|=1} u_{i+\ell}+d_{i} u_{i}, \quad i \in \mathbb{Z}^{m}
$$

with a small positive coupling constant $\varepsilon$ acting on $\ell^{2}$-sequences $u=\left(u_{i}\right)$ of arbitrary dimension $m \geqq 1$. Various examples of potentials $d=\left(d_{i}\right)$ are now known such that this operator has only pure point spectrum and a complete set of exponentially localized eigenvectors. It is our aim to present a general approach to the construction of these examples as well as some new ones. Namely, we construct limit periodic potentials $d$ such that the pure point spectrum is dense in $[0,1]$ in one case, and dense in a Cantor set of measure zero in another case.

We proceed as follows. We write

$$
H=D+\varepsilon \Delta
$$

in the form of a $2 m$-dimensional Jacobi matrix, with $D=\operatorname{diag}(d)$ and

$$
\Delta=\sum_{|\ell|=1}\left(\delta_{i, j+\ell}\right)
$$

where $|\ell|=\sum_{\mu=1}^{m}\left|\ell_{\mu}\right|$ for $\ell=\left(\ell_{1}, \ldots, \ell_{m}\right)$. For a suitably fixed $D$ and sufficiently small $\varepsilon$, we then prove the existence of another diagonal matrix $\hat{D}$ close to $D$ such that

$$
V^{-1}(\hat{D}+\varepsilon \Delta) V=D
$$


with a unitary isomorphism $V$ of $\ell^{2}\left(\mathbb{Z}^{m}\right)$. The new Schrödinger operator $\hat{H}=\hat{D}+\varepsilon \Delta$ is thus unitarily equivalent to the multiplication operator $D$, hence

$$
\sigma_{p p}(\hat{H})=\sigma_{p p}(D)=\left\{d_{i}: i \in \mathbb{Z}^{m}\right\},
$$

and $\sigma(\hat{H})=\operatorname{cl} \sigma_{p p}(\hat{H})$. The corresponding eigenvectors of $\hat{H}$ form a complete set, since they are the columns of $V$.

The matrices $\hat{D}$ and $V$ are constructed simultaneously by means of a Newton-type iteration process. This approach requires the solvability of the commutator equation

$$
[D, W]+P=0
$$

for $W$, where $P$ is assumed to vanish on the main diagonal. The solution of this equation introduces the divisors $d_{i}-d_{j}$, and the crucial condition is that these divisors don't get small too fast as $|i-j|$ gets large so that their influence is overcome by the quadratic convergence of the iterarion scheme;

$$
\left|d_{i}-d_{j}\right|^{-1} \leqq c|i-j|^{\tau}, \quad i \neq j,
$$

with $c>0$, is a typical example of such a small divisor condition. In fact, the problem consists in finding interesting sequences $d=\left(d_{i}\right)$ satisfying such a condition. For instance, it is not at all clear that there are limit periodic sequences of that kind.

What we described so far amounts to solving an inverse problem - we construct a potential for a given spectrum. There is also a solution of the direct problem of determining the spectrum of a given potential, but only under a much more restrictive hypothesis. In order to construct $\hat{D}$ and $V$ with

$$
V^{-1}(D+\varepsilon \Delta) V=\hat{D} \text {, }
$$

we have to be able to solve the linearized equation

$$
[\hat{D}, W]+P=0
$$

for all $\hat{D}$ near $D$. This requires the small divisor conditions to be preserved under small perturbations. This is hardly ever the case but nonetheless there are nontrivial examples of that kind. They yield unbounded discrete Schrödinger operators with a dense pure point spectrum on the real line.

The preceding results are obtained as straightforward corollaries of more general results about arbitrary perturbations $D+P$ of infinite diagonal matrices $D$. These results are formulated in terms of a general translation invariant Banach algebra of $m$-dimensional real sequences. This allows us to obtain different examples of Schrödinger operators with pure point spectrum just by switching frome one Banach algebra to another one.

This note was stimulated by a paper by Craig [4], and its basic ideas are due to him - or rather Rüssmann [7] and Moser [6], as far as the solution of Eq. (0.1) is concerned. We just put them into a general framework and added examples of limit periodic sequences satisfying a small divisor condition. When this work on the inverse problem was essentially completed, we learned of a similar treatment of the direct problem for some nontrivial examples by Bellissard et al. [2]. It turned out that their treatment fitted into our general approach quite easily. 


\section{Main Results}

Let $m \geqq 1$ be an integer. Our starting point is a Banach algebra $\mathscr{M}$ of real $m$-dimensional sequences $a=\left(a_{i}\right)_{i \in \mathbb{Z}^{m}}$ with the operations of pointwise addition and multiplication of sequences. In particular, the constant sequence 1 is supposed to belong to $\mathscr{M}$ and have norm one. In addition, $\mathscr{M}$ is required to be invariant under translations: if $a \in \mathscr{M}$, then $T_{k} a \in \mathscr{M}$ with $\left\|T_{k} a\right\|_{\mathscr{M}}=\|a\|_{\mathscr{M}}$ for all $k \in \mathbb{Z}^{m}$, where $\left(T_{k} a\right)_{i}=a_{i+k}$. In other words, all translations $T_{k}$ are isometries of $\mathscr{M}$.

The translation invariance of $\mathscr{M}$ implies that

$$
\|a\|_{\mathscr{B}} \leqq\|a\|_{\mathscr{M}},
$$

where $\|a\|_{\mathscr{B}}=\sup \left|a_{i}\right|$. In fact, if $a$ were unbounded, then there would exist translates $a^{n}=T_{k_{n}} a, n \geqq 1$, with $n^{-1}\left|a_{0}^{n}\right|=n^{-1}\left|a_{k_{n}}\right| \geqq 1$ and $n^{-1}\left\|a^{n}\right\|_{\mathscr{M}}=n^{-1}\|a\|_{\mathscr{M}} \rightarrow 0$, so in the limit we had a sequence $\alpha \in \mathscr{M}$ with $\|\alpha\|_{\mathscr{M}}=0$ and $\left|\alpha_{0}\right| \geqq 1$. Now, if we had $\|a\|_{\mathscr{B}}$ $>\|a\|_{\mathscr{M}}$, then $1 \pm a /\|a\|_{\mathscr{B}}$ would be invertible in $\mathscr{M}$ which is a contradiction.

The small divisor conditions take the following form. Let $d=\left(d_{i}\right)$ be an arbitrary real sequence. We call $d$ a distal sequence for $\mathscr{M}$ if

$$
\left(d-T_{k} d\right)^{-1} \in \mathscr{M}, \quad\left\|\left(d-T_{k} d\right)^{-1}\right\|_{\mathscr{M}} \leqq \Omega(|k|), \quad 0 \neq k \in \mathbb{Z}^{m},
$$

where $\Omega$ is a continuous function $[0, \infty[\rightarrow[1, \infty[$ with the property that both

$$
\Phi(\sigma)=\sigma^{-4 m} \sup _{r \geqq 0} \Omega(r) e^{-\sigma r}, \quad \sigma \geqq 0
$$

and

$$
\Psi(\sigma)=\inf _{S_{\sigma}} \prod_{\nu=0}^{\infty} \Phi\left(\sigma_{\nu}\right)^{2^{-v-1}}, \quad \sigma \geqq 0
$$

are finite for $\sigma>0$, where $S_{\sigma}$ is the set of all sequences $\sigma_{\sigma} \geqq \sigma_{1} \geqq \ldots \geqq 0$ with $\sum \sigma_{v} \leqq \sigma$. Such functions $\Omega$ are called approximation functions; they were first introduced by Rüssmann [7].

Clearly, if $\Omega$ is multiplied by a positive constant, then $\Phi$ and $\Psi$ are multiplied by the same constant. Moreover, the product of two approximation functions is again an approximation function. In the particular case

$$
\Omega(r)=r^{\tau}, \quad \tau \geqq 0,
$$

one easily finds

$$
\Psi(\sigma) \leqq c \sigma^{-4 m-\tau}
$$

by choosing $\sigma_{v}=\sigma \cdot 2^{-v-1}$, the constant $c$ depending on $\tau$ and the dimension $m$. For a general $\Omega$ there are simple sufficient conditions due to Rüssmann [7] under which $\Phi$ and $\Psi$ are finite. We recall them in an appendix.

Finally, we introduce the space M of all matrices $A=\left(a_{i j}\right)_{i, j \in \mathbb{Z}^{m}}$ satisfying

$$
A_{k}=\left(a_{i, i+k}\right) \in \mathscr{M}, \quad k \in \mathbb{Z}^{m} .
$$

In other words, $A \in \mathrm{M}$ if all diagonals of $A$ belong to $\mathscr{M}$. In $\mathrm{M}$ we define a scale of Banach spaces

$$
\mathrm{M}^{s}=\left\{A \in \mathrm{M}:\|A\|_{s}<\infty\right\}, \quad 0 \leqq s \leqq \infty,
$$


where

$$
\|A\|_{s}=\sup _{k \in \mathbb{Z}^{m}}\left\|A_{k}\right\|_{M} e^{|k| s}, \quad 0 \leqq S \leqq \infty
$$

Clearly,

$$
\mathrm{M}^{s} \supset \mathrm{M}^{t}, \quad\|\cdot\|_{s} \leqq\|\cdot\|_{t}, \quad 0 \leqq s \leqq t \leqq \infty .
$$

In particular, $\mathrm{M}^{\infty}$ is the space of all diagonal matrices in $\mathrm{M}$, and we have an isometry $a \mapsto \operatorname{diag}(a)$ of $\mathscr{M}$ and $\mathrm{M}$.

We are now ready to formulate our main results.

Theorem A. Let $D$ be a diagonal matrix whose diagonal $d$ is a distal sequence for $\mathscr{M}$. Let $0<s \leqq \infty$ and $0<\sigma \leqq 1, \frac{s}{2}$. If $P \in \mathrm{M}^{s}$,

$$
\|P\|_{s} \leqq \delta \cdot \Psi\left(\frac{\sigma}{2}\right)^{-1}
$$

where $\delta>0$ depends on the dimension $m$ only, then there exists another diagonal matrix $\hat{D}$ and an invertible matrix $V$ such that

$$
V^{-1}(\hat{D}+P) V=D \text {. }
$$

In fact, $V, V^{-1} \in \mathrm{M}^{s-\sigma}$ and $\hat{D}-D \in \mathrm{M}^{\infty}$ with

$$
\begin{gathered}
\|V-I\|_{s-\sigma},\left\|V^{-1}-I\right\|_{s-\sigma} \leqq C \cdot\|P\|_{s}, \\
\|\hat{D}-D+[P]\|_{\infty} \leqq C^{2} \cdot\|P\|_{s}^{2},
\end{gathered}
$$

where $C=\delta^{-1} \Psi\left(\frac{\sigma}{2}\right)$, and $[\cdot]$ denotes the canonical projection $\mathrm{M}^{s} \rightarrow \mathrm{M}^{\infty}$. If $P$ is hermitian, then $V$ can be chosen to be unitary on $l^{2}\left(\mathbb{Z}^{m}\right)$.

This theorem allows us to solve the inverse problem of finding a potential for a given spectrum. The direct problem of finding the spectrum of a given potential is somewhat more natural, but, as we already pointed out, requires the much more restrictive hypothesis that the small divisor conditions are preserved under perturbations.

Theorem B. Let $D$ be a diagonal matrix with a diagonal d such that all sequences $\hat{d}$ with $\hat{d}-d \in \mathscr{M},\|\hat{d}-d\|_{\mathscr{M}} \leqq \gamma \leqq 1$ are distal sequences for $\mathscr{M}$ with one and the same approximation function $\Omega$. Let $0<s \leqq \infty$ and $0<\sigma \leqq 1, \frac{S}{2}$. If $P \in \mathrm{M}^{\mathrm{s}}$,

$$
\|P\|_{s} \leqq \gamma \delta \cdot \Psi\left(\frac{\sigma}{2}\right)^{-1},
$$

$\delta$ as in Theorem $A$, then there exist $\hat{D}$ and $V$ with the same properties as in Theorem A so that

$$
V^{-1}(D+P) V=\hat{D} \text {. }
$$

A sequence $d$ with the property described above is called a stable distal sequence for $\mathscr{M}$. 
We postpone the proof of these theorems to the end of this note, and consider first their consequences for discrete Schrödinger operators.

Corollary A. Let $d$ be a distal sequence for some translation invariant Banach algebra $\mathscr{M}$ of $m$-dimensional real sequences. Then, for $0 \leqq \varepsilon \leqq \varepsilon_{0}, \varepsilon_{0}>0$ sufficiently small, there exists a sequence $\hat{d}$ with $\hat{d}-d \in \mathscr{M},\|d-d\|_{\mathscr{M}} \leqq \varepsilon^{2} / \varepsilon_{0}^{2}$, such that the discrete Schrödinger operator

$$
(\hat{H} u)_{i}=\varepsilon \sum_{|\ell|=1} u_{i+\ell}+\hat{d}_{i} u_{i}, \quad i \in \mathbb{Z}^{m}
$$

has pure point spectrum $\left\{d_{i}: i \in \mathbb{Z}^{m}\right\}$ and a complete set of exponentially localized eigenvectors with decay rate $1+\log \frac{\varepsilon_{0}}{\varepsilon}$.

Proof. We apply Theorem A to the Jacobi matrix $D+\varepsilon \Delta, D=\operatorname{diag}(d)$. Clearly, $\Delta \in \mathrm{M}^{s}$ with $\|\Delta\|_{s}=e^{s}$ for all $s \geqq 0$, so for $\sigma=1$ the smallness condition amounts to

$$
\|\varepsilon \Delta\|_{s}=\varepsilon e^{s} \leqq \theta=\delta \cdot \Psi\left(\frac{1}{2}\right)^{-1}
$$

with $s \geqq 2$, thus

$$
0 \leqq \varepsilon \leqq \varepsilon_{0}=e^{-2} \cdot \theta \text {. }
$$

This also bounds $s$ from above by $\log \theta / \varepsilon$. By Theorem A there now exists a unitary matrix $V \in \mathrm{M}^{s-1}$ and a diagonal matrix $\hat{D}=\operatorname{diag}(\hat{d})$ with $\hat{D}-D \in \mathrm{M}^{\infty}$ such that

$$
V^{-1}(\hat{D}+\varepsilon \Delta) V=D \text {. }
$$

But $V$ defines a unitary isomorphism on $\ell^{2}\left(\mathbb{Z}^{m}\right)$ in view of $\|\cdot\|_{\mathscr{B}} \leqq\|\cdot\|_{\mathscr{M}}$ and the exponential decay of the diagonals of $V$ in the norm $\|\cdot\|_{\mathscr{M}}$, so the discrete Schrödinger operator $\hat{H}=\varepsilon \Delta+\hat{D}$ is unitarily equivalent to the multiplication operator $D$. Hence,

$$
\sigma_{p p}(\hat{H})=\sigma_{p p}(D)=\left\{d_{i}: i \in \mathbb{Z}^{m}\right\}
$$

and the corresponding eigenvectors form a complete set. Since they are the columns of $V$, they are exponentially localized with decay rate

$$
s-1=\log \frac{\theta}{e \varepsilon}=1+\log \frac{\varepsilon_{0}}{\varepsilon}
$$

by choosing $s=\log \theta / \varepsilon$. Finally,

$$
\|\hat{d}-d\|_{\mathcal{M}}=\|\hat{D}-D\|_{\infty} \leqq \theta^{-2}\|\varepsilon \Delta\|_{2}^{2}=\varepsilon^{2} / \varepsilon_{0}^{2}
$$

by choosing $s=2$.

In the same manner one proves

Corollary B. If $d$ is a stable distal sequence for some translation invariant Banach algebra $\mathscr{M}$ of $m$-dimensional real sequences, then Corollary $A$ holds with the role of $d$ and $\hat{d}$ interchanged.

The main problem now is to construct interesting examples of distal sequences. This we do in the next two sections, then we prove Theorems A and B. 


\section{Limit Periodic Examples}

Fix $m \geqq 1$, and let $\mathscr{P}$ be the set of all real $m$-dimensional sequences $a=\left(a_{i}\right)$ with period $2^{n}, n \geqq 0$, in each dimension; that is,

$$
a_{i}=a_{j}, i-j \in 2^{n} \mathbb{Z}^{m} .
$$

The closure of $\mathscr{P}$ with respect to the sup norm $\|\cdot\|_{\mathscr{B}}$ is a Banach algebra, which we denote by $\mathscr{L}$. It is a subspace of the space of all limit periodic sequences, which is obtained as the closure of the set of all periodic sequences.

In the following we give two examples of sequences in $\mathscr{L}$, which are distal sequences for $\mathscr{L}$, and whose values lie dense in $[0,1]$ in one case, and dense in a Cantor set $\mathscr{C} \subset[0,1]$ of measure zero in the other case. It follows by Corollary A that there exist limit periodic potentials having a pure point spectrum with the same density properties.

We note that a sequence $d \in \mathscr{L}$ satisfies

$$
\left(d-T_{k} d\right)^{-1} \in \mathscr{L},\left\|\left(d-T_{k} d\right)^{-1}\right\|_{\mathscr{B}} \leqq \Omega(|k|), \quad k \neq 0,
$$

if and only if

$$
\inf _{i \in \mathbb{Z}^{m}}\left|d_{i}-d_{i+k}\right| \geqq \Omega(|k|)^{-1}, \quad k \neq 0 .
$$

The proof is straightforward.

To begin with, let $\alpha_{v}, v \geqq 1$, be the characteristic function of the set

$$
A_{v}= \begin{cases}\bigcup_{N \in \mathbb{Z}}\left[N \cdot 2^{v}, N \cdot 2^{v}+2^{v-1}\right), & v \text { even } \\ \bigcup_{N \in \mathbb{Z}}\left[N \cdot 2^{v}+2^{v-1}, N \cdot 2^{v}+2^{v}\right), & v \text { odd } .\end{cases}
$$

Then $\alpha_{v}$ has period $2^{v}$, and we have the following two lemmas.

Lemma 2.1. For $n \geqq 1$, the map

$$
\left(\alpha_{1}, \ldots, \alpha_{n}\right): \mathbb{Z} \rightarrow\{0,1\}^{n}
$$

has period $2^{n}$, and is one-to-one as a map from $\mathbb{Z} / 2^{n} \mathbb{Z}$ to $\{0,1\}^{n}$.

Proof. The first statement is obvious, and the second statement is easily proven by induction.

Lemma 2.2. Let $n \geqq 1$ and $i, j \in \mathbb{Z}$. If $\alpha_{n+v}(i)=1$ for $v=1,2,3$ and $|i-j|<2^{n}$, then $\alpha_{n+3}(j)=1$.

Proof. By periodicity we may assume that $0 \leqq i<2^{n+3}$. If $n$ is odd, then our assumptions imply $2^{n+1} \leqq i<2^{n+2}-2^{n}$ and thus $0 \leqq j<2^{n+2}$, hence $\alpha_{n+3}(j)=1$. If $n$ is even, then $2^{n+2}+2^{n} \leqq i<2^{n+3}-2^{n+1}$ and thus $2^{n+2} \leqq j<2^{n+3}$, hence $\alpha_{n+3}(j)=1$ also in this case. 
Example 1. The $m$-dimensional sequence $d=\left(d_{i}\right)$, given by

$$
d_{i}=\sum_{\nu=1}^{\infty} \sum_{\mu=1}^{m} \alpha_{\nu}\left(i_{\mu}\right) 2^{-(v-1) m-\mu}, i=\left(i_{1}, \ldots, i_{m}\right) \in \mathbb{Z}^{m},
$$

belongs to $\mathscr{L}$ and lies dense in $[0,1]$. It is a distal sequence for $\mathscr{L}$ with

$$
\left\|\left(d-T_{k} d\right)^{-1}\right\|_{\mathscr{B}} \leqq 16^{m}|k|^{m}, \quad 0 \neq k \in \mathbb{Z}^{m} .
$$

Proof. We first consider the case $m=1$, where

$$
d_{i}=\sum_{v=1}^{\infty} \alpha_{v}(i) 2^{-v}, \quad i \in \mathbb{Z} .
$$

We define sequences $d^{n}=\left(d_{i}^{n}\right), n \geqq 1$, by

$$
d_{i}^{n}=\sum_{\nu=1}^{n} \alpha_{v}(i) 2^{-v} .
$$

By Lemma 2.1, $d^{n}$ has period $2^{n}$ and takes on all values $N \cdot 2^{-n}, 0 \leqq N<2^{n}$. Moreover, $\left\|d-d^{n}\right\|_{\mathscr{B}} \leqq 2^{-n}$. Hence, $d \in \mathscr{L}$, and its values lie dense in $[0,1]$.

To prove (2.1), let $i \neq j$, and fix $n \geqq 1$ so that

$$
2^{n-1} \leqq|i-j|<2^{n} \text {. }
$$

Then $d_{i}^{n} \neq d_{j}^{n}$ and $\left|d_{i}^{n}-d_{j}^{n}\right| \geqq 2^{-n}$ by Lemma 2.1. On the other hand, for $\tilde{d}^{n}=d-d^{n}$ we have

$$
\left|\tilde{d}_{i}^{n}-\tilde{d}_{j}^{n}\right| \leqq \sum_{v=n+1}^{\infty}\left|\alpha_{v}(i)-\alpha_{v}(j)\right| \cdot 2^{-v} \leqq \frac{7}{8} \cdot 2^{-n}
$$

by Lemma 2.2, hence

$$
\left|d_{i}-d_{j}\right| \geqq\left|d_{i}^{n}-d_{j}^{n}\right|-\left|\tilde{d}_{i}^{n}-\tilde{d}_{j}^{n}\right| \geqq \frac{1}{8} \cdot 2^{-n} \geqq \frac{1}{16}|i-j|^{-1}
$$

with (2.2), and (2.1) follows for $m=1$.

The general case $m \geqq 1$ is handled analogously, defining $d^{n}$ by

$$
d_{i}^{n}=\sum_{\nu=1}^{n} \sum_{\mu=1}^{m} \alpha_{\nu}\left(i_{\mu}\right) \cdot 2^{-(v-1) m-\mu} .
$$

Again, assume (2.2). By Lemma 2.1, we have $d_{i}^{n} \neq d_{j}^{n}$ and therefore $\left|d_{i}^{n}-d_{j}^{n}\right| \geqq 2^{-n m}$. On the other hand, Lemma 2.2 implies

$$
\begin{aligned}
\left|\tilde{d}_{i}^{n}-\tilde{d}_{j}^{n}\right| & \leqq \sum_{\nu=n+1}^{\infty} \sum_{\mu=1}^{m}\left|\alpha_{v}\left(i_{\mu}\right)-\alpha_{\nu}\left(j_{\mu}\right)\right| \cdot 2^{-(v-1) m-\mu} \\
& \leqq\left(1-2^{-m}\right)\left(\sum_{\nu=n+1}^{\infty} 2^{-(v-1) m}-2^{-(n+2) m}\right) \\
& \leqq\left(1-2^{-3 m}\right) \cdot 2^{-n m},
\end{aligned}
$$

where $\tilde{d}^{n}=d-d^{n}$ as before. Hence,

$$
\left|d_{i}-d_{j}\right| \geqq 2^{-3 m} \cdot 2^{-n m} \geqq 2^{-4 m} \cdot|i-j|^{-m}
$$

with (2.2), and (2.1) follows for arbitrary $m \geqq 1$. 
Example 2. The $m$-dimensional sequence $d=\left(d_{i}\right)$, given by

$$
d_{i}=2 \sum_{v=1}^{\infty} \sum_{\mu=1}^{m} \alpha_{v}\left(i_{\mu}\right) 3^{-(v-1) m-\mu}, \quad i=\left(i_{1}, \ldots, i_{m}\right) \in \mathbb{Z}^{m},
$$

belongs to $\mathscr{L}$ and is a distal sequence for $\mathscr{L}$ with

$$
\left\|\left(d-T_{k} d\right)^{-1}\right\|_{\mathscr{B}} \leqq 3^{m}|k|^{\lambda m}, \quad 0 \neq k \in \mathbb{Z}^{m},
$$

where $\lambda=\log _{2} 3$. Its values lie dense in the standard Cantor set $\mathscr{C}$ of all real numbers in $[0,1]$ whose triadic expansion consists only of zeroes and twos.

Proof. We prove (2.3). If $2^{n-1} \leqq|i-j|<2^{n}$, and $d^{n}, \tilde{d}^{n}$ are defined as before, then $\left|d_{i}^{n}-d_{j}^{n}\right| \geqq 2 \cdot 3^{-m n}$, while

$$
\left|\tilde{d}_{i}^{n}-\tilde{d}_{j}^{n}\right| \leqq 2 \sum_{v=n+1}^{\infty} \sum_{\mu=1}^{m} 3^{-(v-1) m-\mu}=3^{-m n} .
$$

Thus,

$$
\left|d_{i}-d_{j}\right| \geqq 3^{-m n} \geqq 3^{-m}|i-j|^{-\lambda m}, \quad \lambda=\log _{2} 3,
$$

and (2.3) follows. The remaining statements are easy to prove.

The question arises, how typical such distal sequences in $\mathscr{L}$ are. Here is a first answer.

Proposition 1. For an approximation function $\Omega$ with $\Omega(r) \geqq r^{m}$, the set

$$
\mathscr{D}=\left\{d \in \mathscr{L}: \sup _{k \neq 0}\left\|\left(d-T_{k} d\right)^{-1}\right\|_{\mathscr{B}} \Omega(|k|)^{-1}<\infty\right\}
$$

is of first category and dense in $\mathscr{L}$.

Proof. Clearly, $\mathscr{D}=\bigcup_{N=1}^{\infty} \mathscr{D}_{N}$, where

$$
\mathscr{D}_{N}=\left\{d \in \mathscr{L}:\left\|\left(d-T_{k} d\right)^{-1}\right\|_{\mathscr{B}} \leqq N \cdot \Omega(|k|), k \neq 0\right\} .
$$

Each $\mathscr{D}_{N}$ is closed and nowhere dense, since its complement contains $\mathscr{P}$. So $\mathscr{D}$ is of first category. Now let $a \in \mathscr{P}$, let $d \in \mathscr{L}$ be the sequence of Example 1, and consider the sequence $a^{\varepsilon}=a+\varepsilon d$. Since

$$
\inf _{a_{i} \neq a_{j}}\left|a_{i}-a_{j}\right|=\delta>0,
$$

with the convention, say, $\delta=1$ for a constant sequence $a$, one easily finds

$$
\left|a_{i}^{\varepsilon}-a_{j}^{\varepsilon}\right| \geqq \varepsilon \cdot 16^{-m}|i-j|^{-m}, i \neq j, 0 \leqq \varepsilon \leqq \delta / 2,
$$

by distinguishing the cases $a_{i}=a_{j}$ and $a_{i} \neq a_{j}$. Hence, for $\varepsilon \neq 0$,

$$
\left\|\left(a^{\varepsilon}-T_{k} a^{\varepsilon}\right)^{-1}\right\|_{\mathscr{B}} \leqq 16^{m} \varepsilon^{-1} \Omega(|k|),
$$

and $a^{\varepsilon} \in \mathscr{D}$. It follows that $\mathscr{D}$ is dense in $\overline{\mathscr{P}}=\mathscr{L}$.

This proposition shows that our examples are not typical in the sense of Baire category. Our first example is also not typical from another point of view. 
Proposition 2. The set

$$
\mathscr{T}=\{d \in \mathscr{L}: d \text { is dense in some interval }\}
$$

is of first category and dense in $\mathscr{L}$.

Proof. We have $\mathscr{T}=\bigcup_{a, b \text { rational, } a<b} \mathscr{T}_{a, b}$, where, for fixed $a<b$,

$$
\mathscr{T}_{a, b}=\{d \in \mathscr{L}: d \text { dense in }[a, b]\} .
$$

$\mathscr{T}_{a, b}$ is easily seen to be closed, and it is nowhere dense, since its complement contains $\mathscr{P}$. So $\mathscr{T}$ is of first category. The sequence $a^{\varepsilon}=a+\varepsilon d, \varepsilon \neq 0$, introduced in the preceding proof, is dense in some interval, since otherwise $d$ would be nowhere dense. Consequently, $\mathscr{T}$ is dense in $\mathscr{L}$.

For a similar result in the continuous case see [1].

\section{Other Examples}

A couple of known examples of discrete Schrödinger operators with pure point spectrum is contained in

Corollary C. Let $\mathscr{F}$ be a translation invariant Banach algebra of functions defined on $\mathbb{R}$, and let $\omega \in \mathbb{R}^{m}, m \geqq 1$. If $\phi$ is an arbitrary function satisfying

$$
\left(\phi-T_{k \omega} \phi\right)^{-1} \in \mathscr{F},\left\|\left(\phi-T_{k \omega} \phi\right)^{-1}\right\|_{\mathscr{F}} \leqq \Omega(|k|), k \neq 0
$$

with some approximation function $\Omega$, then, for $0 \leqq \varepsilon \leqq \varepsilon_{0}, \varepsilon_{0}>0$ sufficiently small, there exists a function $\hat{\phi}$ with $\hat{\phi}-\phi \in \mathscr{F},\|\hat{\phi}-\phi\|_{\mathscr{F}} \leqq \varepsilon^{2} / \varepsilon_{0}^{2}$, such that the discrete Schrödinger operator

$$
(\hat{H} u)_{i}=\varepsilon \sum_{|\ell|=1} u_{i+\ell}+\hat{\phi}(i \omega) \cdot u_{i}, i \in \mathbb{Z}^{m}
$$

has pure point spectrum $\left\{\phi(i \omega), i \in \mathbb{Z}^{m}\right\}$ and a complete set of exponentially localized eigenvectors with decay rate $1+\log \varepsilon_{0} / \varepsilon$.

If condition (3.1) even holds for all functions $\psi$ with $\psi-\phi \in \mathscr{F},\|\psi-\phi\|_{\mathscr{F}} \leqq \gamma$, $\gamma>0$, then the preceding statement is also true with the role of $\phi$ and $\hat{\phi}$ interchanged.

Above, we used the notation $k \omega=\sum_{\mu=1}^{m} k_{\mu} \omega_{\mu}$ and $T_{k \omega} \phi=\phi(\cdot+k \omega)$. We stress that our assumptions include that $\|f\|_{\mathscr{F}}=0$ implies $f=0$ everywhere.

Proof. Let $\mathscr{M}$ be the set of all real $m$-dimensional sequences $a=\left(a_{i}\right)$ satisfying $a_{i}=f(i \omega)$ for some $f \in \mathscr{F}$, and set

$$
\|a\|_{\mathscr{M}}=\inf \|f\|_{\mathscr{F}}
$$

taking the inf over all $f \in \mathscr{F}$ with this property. Since $\|f\|_{\infty} \leqq\|f\|_{\mathscr{F}}$ for all $f \in \mathscr{F}$ by the same arguments as for sequences, $\|\cdot\|_{\mathscr{M}}$ is a norm on $\mathscr{M}$, and $\mathscr{M}$ is a translation invariant Banach algebra with this norm. Now it is easy to see that the sequence $d$, given by $d_{i}=\phi(i \omega)$, is a distal sequence for $\mathscr{M}$ with $\left\|\left(d-T_{k} d\right)^{-1}\right\|_{\mathscr{M}} \leqq \Omega(|k|)$ by (3.1). An application of Corollaries $\mathrm{A}$ and $\mathrm{B}$ then gives the result. 
We give two applications of Corollary C. In both cases, $\omega$ is required to satisfy a diophantine condition

$$
\|k \omega\| \geqq \Omega(|k|)^{-1}, \quad k \neq 0,
$$

where $\|x\|=\min _{n \in \mathbb{Z}}|x+n|$ for $x \in \mathbb{R}$. Apart from trivial cases, such a condition is necessary to verify (3.1). For $m>1$, it incidentally implies that the points $i \omega, i \in \mathbb{Z}^{m}$, lie dense on the real line.

As a first example, we consider the space $\mathscr{V}$ of all real functions of period 1 and bounded variation. With the norm

$$
\|f\|_{\mathscr{V}}=\|f\|_{\infty}+|f|_{\text {total variation }}
$$

$\mathscr{V}$ is a translation invariant Banach algebra. The periodic extension $\phi$ of the identity function on $[0,1]$ belongs to $\mathscr{V}$ and satisfies

$$
\left|\phi-T_{k \omega} \phi\right| \geqq\|k \omega\| \geqq \Omega(|k|)^{-1}, \quad k \neq 0,
$$

by (3.3). From this one obtains $\left(\phi-T_{k \omega} \phi\right)^{-1} \in \mathscr{V}$ with

$$
\left\|\left(\phi-T_{k \omega} \phi\right)^{-1}\right\|_{\mathscr{V}} \leqq 3 \cdot \Omega(|k|)^{2}, \quad k \neq 0 .
$$

So Corollary C applies, and for small positive $\varepsilon$ there exist $\hat{\phi} \in \mathscr{V}$ such that (3.2) has pure point spectrum $\phi(i \omega)$, which is dense in $[0,1]$. This is Craig's example [4].

It is worth mentioning that (3.4) can not hold for a continuous, 1-periodic function $\phi$, since for any $k \omega \notin \mathbb{Z}$ there exists $x$ with $\phi(x)=\phi(x+k \omega)$ by the mean value theorem.

As a second example, we let $r>0$, and consider the space $\mathscr{H}_{r}$ of all real analytic functions on $S_{r}=\{z \in \mathbb{C}:|\operatorname{Im} z|<r\}$ with period 1 and bounded derivative. With the norm

$$
\|f\|_{\mathscr{H}_{r}}=\sup _{z \in S_{r}}|f(z)|+\sup _{z \in S_{r}}\left|f^{\prime}(z)\right|,
$$

this is a translation invariant Banach algebra. The function $\phi=\tan \pi z$ is real meromorphic on $S_{r}$, of period 1 , and satisfies

$$
\left(\phi-T_{x} \phi\right)(z)=-\frac{\sin \pi x}{\cos \pi z \cos \pi(z+x)} .
$$

For $z \in S_{r}$ and $x \in \mathbb{R}$, we have

$$
|\sin \pi x| \geqq 2 \cdot\|x\|,|\cos \pi z \cos \pi(z+x)| \leqq c_{r}=\cosh 2 \pi r .
$$

Together with

$$
\left|f-T_{x} f\right|_{S_{r}} \leqq\|f\|_{\mathscr{H}_{r}} \cdot\|x\|, \quad f \in \mathscr{H}_{r}, x \in \mathbb{R},
$$

and assumption (3.3), this implies

$$
\left(\psi-T_{k \omega} \psi\right)^{-1} \in \mathscr{H}_{r},\left\|\left(\psi-T_{k \omega} \psi\right)^{-1}\right\|_{\mathscr{H}_{r}} \leqq 4 \pi c_{r} \Omega(|k|)^{2}, \quad k \neq 0,
$$

for all $\psi$ with $\psi-\phi \in \mathscr{H}_{r},\|\psi-\phi\|_{\mathscr{H}_{r}} \leqq c_{r}^{-1}$. It follows by Corollary $\mathrm{C}$ that for small positive $\varepsilon$ the discrete Schrödinger operator

$$
(H u)_{i}=\varepsilon \sum_{|\ell|=1} u_{i+\ell}+\phi(i \omega) \cdot u_{i}, \quad i \in \mathbb{Z}^{m}
$$


has pure point spectrum $\{\hat{\phi}(i \omega)\}, \hat{\phi}-\phi \in \mathscr{H}_{r}$ small, which is dense on the real line. This is the example of Fishman et al. [5] for sufficiently small positive $\varepsilon$. In fact, in this case, our result is slightly better, since in addition we obtain a complete set of eigenfunctions.

Fishman, Grempel and Prange actually approached the tangent potential differently. It was observed by Bellissard et al. [2] that perturbation methods are applicable. See [2] for a treatment in a more general setting.

Another example also contained in Corollary $\mathrm{C}$ for sufficiently small $\varepsilon$ can be found in [8].

\section{Proof of Theorem $A$ and $B$}

We outline the proof of Theorem A. We write

$$
\hat{D}-D=\sum_{v=0}^{\infty} A_{v}
$$

as a sum of diagonal matrices $A_{v}$ to be constructed consecutively, and set

$$
D_{n}=\sum_{v=n}^{\infty} A_{v}, \quad n \geqq 0 .
$$

We suppose that at the $n^{\text {th }}$ step of the iteration, we have

$$
V_{n}^{-1}(\hat{D}+P) V_{n}=D+P_{n}+V_{n}^{-1} D_{n} V_{n},
$$

where $V_{n}$ is the product of all transformations applied so far, and $P_{n}$ is small; for $n=0$,

$$
V_{0}=I, P_{0}=P \text {. }
$$

The next transformation $W_{n}$ is then determined by the commutator equation

$$
\left[D, W_{n}\right]+P_{n}+V_{n}^{-1} A_{n} V_{n}=0,
$$

which in addition determines $A_{n}$ by the requirement that $P_{n}+V_{n}^{-1} A_{n} V_{n}$ vanishes on the main diagonal. Solving this equation and setting

$$
V_{n+1}=V_{n} W_{n}, P_{n+1}=W_{n}^{-1}\left(P_{n}+V_{n}^{-1} A_{n} V_{n}\right)\left(W_{n}-I\right)
$$

we obtain

$$
V_{n+1}^{-1}(\hat{D}+P) V_{n+1}=D+P_{n+1}+V_{n+1}^{-1} D_{n+1} V_{n+1}
$$

with a smaller $P_{n+1}$. This completes one step of the iteration. In the limit, we have $P_{n} \rightarrow 0, D_{n} \rightarrow 0$ and $V_{n} \rightarrow V$, hence

$$
V^{-1}(\hat{D}+P) V=D
$$

as wanted.

In this scheme, it turns out, small divisors not only enter with the solution of the linearized equation (4.1) as usual, but also with each product of two matrices involving infinite sums. So we deal with small divisors even in cases where the 
commutator $[D, \cdot]$ is boundedly invertible on its range. In any event, their influence is overcome by the quadratic convergence of the iteration scheme.

We now supply the necessary details, beginning with a couple of lemmas.

Lemma 4.1. If $X \in \mathrm{M}^{s-\sigma}, Y \in \mathrm{M}^{s}$ and $0<\sigma \leqq s$, then $X Y \in \mathrm{M}^{s-\sigma}$,

$$
\|X Y\|_{s-\sigma} \leqq \frac{b}{\sigma^{m}}\|X\|_{s-\sigma}\|Y\|_{s}, \quad \sigma \leqq 1,
$$

the constant $b \geqq 1$ depending only on the dimension $m$.

Proof. It suffices to prove this when $\|X\|_{s-\sigma}=1,\|Y\|_{s}=1$. The diagonals of $Z=X Y$ are

$$
Z_{k}=\sum_{\ell \in \mathbb{Z}^{m}} X_{\ell} \cdot T_{\ell} Y_{k-\ell}
$$

Since

$$
\begin{aligned}
\left\|Z_{k}\right\|_{\mathscr{M}} & \leqq \sum_{\ell \in \mathbb{Z}^{m}}\left\|X_{\ell} \cdot T_{\ell} Y_{k-\ell}\right\|_{\mathcal{M}} \leqq \sum_{\ell \in \mathbb{Z}^{m}}\left\|X_{\ell}\right\|_{\mathcal{M}}\left\|Y_{k-\ell}\right\|_{\mathcal{M}} \\
& \leqq \sum_{\ell \in \mathbb{Z}^{m}} e^{-|\ell| \cdot(s-\sigma)} e^{-|k-\ell| s} \\
& \leqq e^{-|k|(s-\sigma)} \cdot \sum_{\ell \in \mathbb{Z}^{m}} e^{-|\ell| \sigma}
\end{aligned}
$$

converges, we have $Z \in \mathrm{M}^{s-\sigma}$, and

$$
\|Z\|_{s-\sigma} \leqq \sum_{\ell \in \mathbb{Z}^{m}} e^{-|\ell| \sigma} \leqq \frac{b}{\sigma^{m}}, \quad 0<\sigma \leqq 1,
$$

by a simple integration.

Lemma 4.2. If $X \in \mathrm{M}^{s}$ and $\|X-I\|_{s}<\sigma^{m} / b$ for $0<\sigma \leqq 1$, $s$, where $b$ is the constant of Lemma 4.1, then $X$ is invertible in $\mathrm{M}^{s-\sigma}$ with

$$
\left\|X^{-1}-I\right\|_{s-\sigma} \leqq\left(1-b \sigma^{-m}\|X-I\|_{s}\right)^{-1}\|X-I\|_{s} .
$$

Proof. Write down the Neumann series for $X^{-1}$ and use $\left\|(X-I)^{v}\right\|_{s-\sigma}$ $\leqq\left(b \sigma^{-m}\right)^{v-1}\|X-I\|_{s}$, which follows from Lemma 4.1.

Lemma 4.3. If $V, V^{-1} \in \mathrm{M}^{\sigma}$ with $\|V-I\|_{\sigma},\left\|V^{-1}-I\right\|_{\sigma} \leqq \sigma^{m} / 2 b$ for $0<\sigma \leqq 1$, then the equation $\left[P+V^{-1} A V\right]=0$ has a unique solution $A \in \mathrm{M}^{\infty}$ for any $P \in \mathrm{M}$, with $\|A\|_{\infty} \leqq 2 \cdot\|[P]\|_{\infty}$.

Proof. Since $A-\left[V^{-1} A V\right]=\left[\left(V^{-1}-I\right)[V-I, A]\right]$ and hence

$$
\begin{aligned}
\left\|A-\left[V^{-1} A V\right]\right\|_{\infty} & \leqq\left\|\left(V^{-1}-I\right)[V-I, A]\right\|_{0} \\
& \leqq \frac{2 b^{2}}{\sigma^{2 m}}\left\|V^{-1}-I\right\|_{\sigma}\|V-I\|_{\sigma}\|A\|_{\infty} \leqq \frac{1}{2} \cdot\|A\|_{\infty}
\end{aligned}
$$

for $A \in \mathrm{M}^{\infty}$ by Lemma 4.1, the map $A \mapsto A-\left[V^{-1} A V+P\right]$ is a $\frac{1}{2}$-contraction in $\mathrm{M}^{\infty}$ for each $P \in \mathrm{M}$, whose unique fixed point solves $\left[V^{-1} A V+P\right]=0$. 
Lemma 4.4. Let $D$ be a diagonal matrix whose diagonal $d$ is a distal sequence for $\mathscr{M}$. Then the equation $[D, W]+P-[P]=0, P \in \mathrm{M}$, has a unique solution $W \in \mathrm{M}$ with $[W]=0$. If $P \in \mathrm{M}^{s}$ and $0<\sigma \leqq s$, then $W \in \mathrm{M}^{s-\sigma}$ with

$$
\|W\|_{s-\sigma} \leqq \phi(\sigma) \cdot\|P\|_{s},
$$

where $\phi(\sigma)=\sup _{r \geqq 0} \Omega(r) e^{-\sigma r}=\sigma^{-4 m} \Phi(\sigma)$.

Proof. The unique solution $W$ is given by

$$
W_{k}=\left\{\begin{array}{ll}
0, & k=0 \\
\left(d-T_{k} d\right)^{-1} P_{k}, & k \neq 0
\end{array} .\right.
$$

By our assumptions, $W_{k} \in \mathscr{M}$ for all $k$ and hence $W \in \mathrm{M}$. If $P \in \mathrm{M}^{s}$ and $0<\sigma \leqq s$, then

$$
\left\|W_{k}\right\|_{\mathscr{M}} \leqq \Omega(|k|) \cdot e^{-|k| s} \cdot\|P\|_{s} \leqq e^{-(s-\sigma)|k|} \cdot \phi(\sigma) \cdot\|P\|_{s},
$$

and the last statement follows.

Now the iteration scheme. By hypotheses, we are given a diagonal matrix $D$, whose diagonal is a distal sequence for $\mathscr{M}$, and a perturbation $P \in \mathrm{M}^{s}$ with

$$
\|P\|_{s} \leqq \delta \cdot \Psi\left(\frac{\sigma}{2}\right)^{-1}, 0<\sigma \leqq 1, \frac{s}{2} .
$$

As is proven in the appendix, there exists a positive, nonincreasing sequence $\left(\sigma_{v}\right)$ such that

$$
\Psi\left(\frac{\sigma}{2}\right)=\prod_{\nu=0}^{\infty} \Phi_{v}^{2^{-v-1}}, \sum_{v=0}^{\infty} \sigma_{v}=\frac{\sigma}{2},
$$

where $\Phi_{v}=\Phi\left(\sigma_{v}\right)$. We fix such a sequence and set

$$
s_{n}=s-2 \sum_{\nu=0}^{n-1} \sigma_{v}, \quad n \geqq 0,
$$

so that $s=s_{0}>s_{1}>\ldots>s_{n} \rightarrow s-\sigma$. Furthermore, we set

$$
\theta_{n}=c^{1-2^{-n}}\|P\|_{s} \prod_{v=0}^{n-1} \Phi_{v}^{2^{-v-1}}, \quad n \geqq 0
$$

with $c=96 b^{6}$. Then $\theta_{\infty}=c\|P\|_{s} \Psi\left(\frac{\sigma}{2}\right) \leqq c \delta$ by hypothesis, and we choose $\delta$ so that

$$
\theta_{\infty} \leqq c \delta=\frac{1}{3 b} \text {. }
$$

We observe that

$$
c \Phi_{n} \theta_{n}^{2^{n}} \leqq \theta_{\infty}^{2^{n}}
$$

since $\Phi_{v}$ increases with $v$, hence $\Phi_{n}=\prod_{v=n}^{\infty} \Phi_{n}^{2^{n-v-1}} \leqq \prod_{v=n}^{\infty} \Phi_{v}^{2^{n-v-1}}$. 
We now prove inductively that for each $n \geqq 0$ there is $P_{n} \in \mathrm{M}^{s_{n}}$,

$$
\left\|P_{n}\right\|_{s_{n}} \leqq \theta_{n}^{2^{n}}
$$

and an invertible $V_{n} \in \mathrm{M}^{s_{n}+\sigma_{n}}$ with $V_{n}^{-1} \in \mathrm{M}^{s_{n}}$,

$$
\left\|V_{n}-V_{n-1}\right\|_{s_{n}+\sigma_{n}},\left\|V_{n}^{-1}-V_{n-1}^{-1}\right\|_{s_{n}} \leqq \sigma^{2 m} \theta_{\infty}^{2^{n-1}}, \quad n \geqq 1,
$$

such that $V_{n}^{-1}(\hat{D}+P) V_{n}=D+P_{n}+V_{n}^{-1} D_{n} V_{n}$.

This is certainly true for $n=0$, so we assume it holds for the first $n$ indices. This implies

$$
\left\|V_{n}-I\right\|_{s_{n}+\sigma_{n}},\left\|V_{n}^{-1}-I\right\|_{s_{n}} \leqq \sigma^{2 m} \frac{\theta_{\infty}}{1-\theta_{\infty}} \leqq \frac{\sigma^{2 m}}{2 b}
$$

by (4.2), and in particular

$$
\left\|V_{n}\right\|_{s_{n}+\sigma_{n}},\left\|V_{n}^{-1}\right\|_{s_{n}} \leqq \frac{3}{2} .
$$

Since $s_{n}+\sigma_{n}>s_{n}>s-\sigma \geqq \sigma$ and $\sigma \leqq 1$ by assumption, we may apply Lemma 4.3 to obtain $A_{n} \in \mathrm{M}^{\infty}$,

$$
\left\|A_{n}\right\|_{\infty} \leqq 2 \cdot\left\|\left[P_{n}\right]\right\|_{\infty} \leqq 2 \cdot \theta_{n}^{2^{n}}
$$

such that $Q_{n}=P_{n}+V_{n}^{-1} A_{n} V_{n}$ satisfies $\left[Q_{n}\right]=0$. Clearly, $Q_{n} \in \mathrm{M}^{s_{n}}$, and

$$
\left\|Q_{n}\right\|_{s_{n}} \leqq\left\|P_{n}\right\|_{s_{n}}+\frac{3 b^{2}}{\sigma_{n}^{m}}\|A\|_{\infty} \leqq \frac{c^{\prime}}{\sigma_{n}^{m}} \theta_{n}^{2^{n}}, \quad c^{\prime}=8 b^{2}
$$

with Lemma 4.1 and the last estimates.

By Lemma 4.4, we now have a solution $W_{n} \in \mathrm{M}^{s_{n}-\sigma_{n}}$ of $\left[D, W_{n}-I\right]+Q_{n}=0$ satisfying

$$
\left\|W_{n}-I\right\|_{s_{n}-\sigma_{n}} \leqq \phi\left(\sigma_{n}\right) \cdot\left\|Q_{n}\right\|_{s_{n}} \leqq c^{\prime} \sigma_{n}^{3 m} \Phi_{n} \theta_{n}^{2^{n}}
$$

by the preceding estimates and the definition of $\Phi_{n}$. In particular,

$$
\left\|W_{n}-I\right\|_{s_{n}-\sigma_{n}} \leqq \sigma_{n}^{3 m} \theta_{\infty}^{2^{n}} / 12 b \leqq \sigma_{n}^{m} / 3 b
$$

because of $c^{\prime} \leqq c / 12 b$ and (4.3), so $W_{n}$ is invertible in $\mathrm{M}^{s_{n}-2 \sigma_{n}}$,

$$
\left\|W_{n}^{-1}-I\right\|_{s_{n}-2 \sigma_{n}} \leqq \frac{3}{2} \cdot\left\|W_{n}-I\right\|_{s_{n}-\sigma_{n}} \leqq c^{\prime \prime} \sigma_{n}^{3 m} \Phi_{n} \theta_{n}^{2^{n}}, \quad c^{\prime \prime}=12 b^{2}
$$

by Lemma 4.2 .

Now set $V_{n+1}=V_{n} W_{n}, V_{n+1}^{-1}=W_{n}^{-1} V_{n}^{-1}$. Since

$$
s_{n+1}=s_{n}-2 \sigma_{n}, S_{n+1}+\sigma_{n+1} \leqq s_{n}-\sigma_{n}
$$

by construction, we have $V_{n+1} \in \mathrm{M}^{s_{n+1}+\sigma_{n+1}}$ and $V_{n+1}^{-1} \in \mathrm{M}^{s_{n+1}}$. Moreover,

$$
\begin{aligned}
\left\|V_{n+1}-V_{n}\right\|_{s_{n+1}+\sigma_{n+1}} & \leqq\left\|V_{n}\left(W_{n}-I\right)\right\|_{s_{n}-\sigma_{n}} \\
& \leqq \frac{b}{\sigma_{n}^{m}}\left\|V_{n}\right\|_{s_{n}+\sigma_{n}}\left\|W_{n}-I\right\|_{s_{n}-\sigma_{n}} \leqq \sigma^{2 m} \theta_{\infty}^{2^{n}}
\end{aligned}
$$

by (4.4) and (4.8), and similarly for $\left\|V_{n+1}^{-1}-V_{n}^{-1}\right\|_{s_{n+1}}$. 
Finally, $\quad P_{n+1}=W_{n}^{-1} Q_{n}\left(W_{n}-I\right)$ obviously is in $\mathrm{M}^{s_{n+1}}$, and since $\left\|W_{n}^{-1}\right\|_{s_{n}-2 \sigma_{n}} \leqq \frac{3}{2}$ by (4.8) and (4.9), we obtain

$$
\begin{aligned}
\left\|P_{n+1}\right\|_{s_{n+1}} & \leqq \frac{b^{2}}{\sigma_{n}^{2 m}}\left\|W_{n}^{-1}\right\|_{s_{n}-2 \sigma_{n}}\left\|Q_{n}\right\|_{s_{n}}\left\|W_{n}-I\right\|_{s_{n}-\sigma_{n}} \\
& \leqq c \Phi_{n} \theta_{n}^{2^{n+1}} \\
& =\theta_{n+1}^{2^{n+1}}
\end{aligned}
$$

using (4.6), (4.7) and $c=96 b^{6}$. This completes the induction.

The convergence of this scheme presents no problems. For instance, since $A_{0}=-[P]$,

$$
\|\hat{D}-D+[P]\|_{\infty} \leqq \sum_{\nu=1}^{\infty}\left\|A_{v}\right\|_{\infty} \leqq \frac{2}{1-\theta_{\infty}} \cdot \theta_{\infty}^{2} \leqq 3 \cdot \theta_{\infty}^{2}
$$

by (4.5) and (4.2), which gives the desired estimate.

If $P$ is hermitian, then $V$ is not necessarily unitary. But by the simplicity of the eigenvalues, $V^{*} V$ is a diagonal matrix close to the identity, and it suffices to replace $V$ by $V\left(V^{*} V\right)^{-1 / 2}$. This completes the proof of Theorem A.

The proof of Theorem B is just a variant of the preceding proof. Here we suppose we have

$$
V_{n}^{-1}(D+P) V_{n}=\hat{D}_{n}+P_{n}
$$

at the $n^{\text {th }}$ step of the iteration with $P_{n}$ and $\hat{D}_{n}-D$ small. Setting

$$
\hat{D}_{n+1}=\hat{D}_{n}+\left[P_{n}\right] \text {, }
$$

we then solve

$$
\left[\hat{D}_{n+1}, W_{n}-I\right]+P_{n}-\left[P_{n}\right]=0,
$$

and with

$$
V_{n+1}=V_{n} W_{n}, P_{n+1}=W_{n}^{-1}\left(P_{n}-\left[P_{n}\right]\right)\left(W_{n}-I\right)
$$

the $n^{\text {th }}$ step of the iteration is completed. In the limit, $P_{n} \rightarrow 0, \hat{D}_{n} \rightarrow \hat{D}$ and $V_{n} \rightarrow V$, and we obtain the desired result.

To this scheme exactly the same estimates as before - and even better ones apply, where now $Q_{n}=P_{n}-\left[P_{n}\right]$. We just have to observe that

$$
\left\|\hat{D}_{n}-D\right\|_{\infty} \leqq \sum_{\nu=0}^{\infty}\left\|\left[P_{v}\right]\right\|_{\infty} \leqq 2 \cdot \theta_{\infty}
$$

and

$$
\theta_{\infty}=c\|P\|_{s} \psi\left(\frac{\sigma}{2}\right) \leqq c \gamma \delta \leqq \gamma / 2
$$

so that, by the hypothesis of Theorem $\mathrm{B}$, the diagonals of all $\hat{D}_{n}$ are distal sequences for $\mathscr{M}$ with one and the same approximation function. Hence, Lemma 4.4 applies to the equation $\left[\hat{D}_{n+1}, W_{n}-I\right]+Q_{n}=0$ for all $n \geqq 0$. 


\section{Appendix. Approximation Functions}

Let $\Omega$ be a continuous function $[0, \infty[\rightarrow[1, \infty[$, and set

$$
\Phi(\sigma)=\sigma^{-\lambda} \sup _{r \geqq 0} \Omega(r) e^{-\sigma r}, \quad \sigma \geqq 0
$$

and

$$
\Psi(\sigma)=\inf _{S_{\sigma}} \prod_{v=0}^{\infty} \Phi\left(\sigma_{v}\right)^{2^{-v-1}}, \quad \sigma \geqq 0,
$$

where $\lambda \geqq 0$ is fixed, and $S_{\sigma}$ is the set of all sequences $\sigma_{0} \geqq \sigma_{1} \geqq \ldots \geqq 0$ with $\sum \sigma_{v} \leqq \sigma$. Here $\Phi$ and $\Psi$ are allowed to be infinite. They are decreasing functions of $\sigma$ and satisfy $\Phi(\sigma) \leqq \Psi(\sigma)$, as is easily verified.

The set $S_{\sigma}$ is compact with respect to the topology of pointwise convergence, and since

$$
\prod_{v=0}^{\infty} \Phi\left(\sigma_{v}\right)^{2^{-v-1}}=\sup _{n \operatorname{large}} \prod_{v=0}^{n} \Phi\left(\sigma_{v}\right)^{2-v-1}
$$

on accont of $\Phi(\sigma) \geqq 1$ for $\sigma \leqq 1$, the map from $S_{\sigma}$ to the infinite product on the left is lower semicontinuous in this topology. Hence, there is a sequence $\hat{\sigma}_{0} \geqq \hat{\sigma}_{1} \geqq \ldots \geqq 0$ such that

$$
\Psi(\sigma)=\prod_{\nu=0}^{\infty} \Phi\left(\hat{\sigma}_{v}\right)^{-v-1} .
$$

Clearly, $\hat{\sigma}_{v}>0$ for $v \geqq 0$ and $\sum \hat{\sigma}_{v}=\sigma$, if $\Psi(\sigma)$ is finite. (We took this argument from Bellissard et al. [3].)

Following Rüssmann [7] we now show that $\Psi(\sigma)$ is finite for positive $\sigma$, if $\omega(r)$ $=\log \Omega(r)$ satisfies

$$
0 \leqq \omega(r) \leqq \omega\left(r^{\prime}\right), \quad 0 \leqq r \leqq r^{\prime}
$$

and

$$
\frac{\omega(r)}{r}>\frac{\omega\left(r^{\prime}\right)}{r^{\prime}} \rightarrow 0, \quad 0 \leqq r<r^{\prime} \rightarrow \infty
$$

and furthermore $\int^{\infty} r^{-2} \omega(r) d r<\infty$. More specifically, we prove

Lemma A.1. If $\omega(r)=\log \Omega(r)$ satisfies the preceding conditions, and

$$
\frac{1}{\log 2} \int_{s}^{\infty} r^{-2} \omega(r) d r \leqq \sigma,
$$

then

$$
\Psi(\sigma) \leqq(4 s / \omega(s))^{\lambda} e^{\sigma s}
$$

Proof. Set

$$
r_{v}=2^{v+1} \cdot s, \sigma_{v}=\frac{\omega\left(r_{v}\right)}{r_{v}}, \quad v \geqq 0 .
$$


Then $\sigma_{0}>\sigma_{1}>\ldots>0$ by (A.2), and

$$
\sum_{v=0}^{\infty} \sigma_{v} \leqq \int_{-1}^{\infty} \frac{\omega\left(r_{v}\right)}{r_{v}} d v \leqq \frac{1}{\log 2} \int_{s}^{\infty} r^{-2} \omega(r) d r \leqq \sigma
$$

by assumption. So this sequence belongs to $S_{\sigma}$. Next, $\omega(r)-\sigma_{v} r \leqq 0$ for $r \geqq r_{v}$ again by (A.2), hence

$$
\sup _{0 \leqq r<\infty} \omega(r)-\sigma_{v} r=\sup _{0 \leqq r \leqq r_{v}} \omega(r)-\sigma_{v} r \leqq \omega\left(r_{v}\right)
$$

with (A.1), and we obtain

$$
\Phi\left(\sigma_{v}\right) \leqq \sigma_{v}{ }^{-\lambda} e^{\omega\left(r_{v}\right)}=\left(r_{v} / \omega\left(r_{v}\right)\right)^{\lambda} e^{r_{v}, \sigma_{v}} .
$$

Since

$$
\prod_{v=0}^{\infty} r_{v}^{2^{-v-1}}=4 s
$$

and $\omega\left(r_{v}\right) \geqq \omega(s)$ by $($ A.1), we arrive at

$$
\Psi(\sigma) \leqq \prod_{\nu=0}^{\infty} \Phi\left(\sigma_{v}\right)^{2^{-v-1}} \leqq(4 s / \omega(s))^{\lambda} e^{\sigma s} .
$$

This lemma allows us to estimate $\Psi(\sigma)$ quite easily. For example, if $\Omega(r)=e^{r \bar{r}}$, then

$$
s=a \sigma^{-2}, \quad a=4 / \log ^{2} 2
$$

satisfies the hypothesis of the lemma, and $\Psi(\sigma) \leqq a \sigma^{-\lambda} e^{a / \sigma}$ follows. For a more subtle example, see Rüssmann [7].

Acknowledgement. I would like to thank Walter Craig, Jürgen Moser, and Barry Simon for helpful discussions.

\section{References}

1. Avron, J., Simon, B. : Almost periodic Schrödinger operators. I. Commun. Math. Phys. 82, 101-120 (1981)

2. Bellissard, J., Lima, R., Scoppola, E.: Localization in v-dimensional incommensurate structures. Commun. Math. Phys. 88, 465-477 (1983)

3. Bellissard, J., Lima, R., Testard, D.: A metal-insulator transition for the almost Mathieu model. Commun. Math. Phys. 88, 207-234 (1983)

4. Craig, W.: Pure point spectrum for discrete almost periodic Schrödinger operators. Commun. Math. Phys. 88, 113-131 (1983)

5. Fishman, S., Grempel, D.R., Prange, R.E.: Preprint, University of Maryland (1982)

6. Moser, J.: Convergent series expansions for quasi-periodic motions. Math. Ann. 169, 136-176(1967)

7. Rüssmann, H.: On the one-dimensional Schrödinger equation with a quasi-periodic potential. Ann. New York Acad. Sci. 357, 90-107 (1980)

8. Sarnak, P.: Spectral behaviour of quasi-periodic potentials. Commun. Math. Phys. 84, 377-401 (1982)

Communicated by B. Simon

Received September 7, 1982; in revised form November 29, 1982 
